\title{
Resección artroscópica del polo distal del escafoides en la artrosis escafo-trapecio-trapezoidea
}

\author{
J. Casañas Sintes \\ Traumaunit. Centro Médico Teknon. Barcelona. España.
}

\begin{abstract}
Resumen: La artrosis escafo-trapecio-trapezoidea se asocia frecuentemente a una inestabilidad carpiana. En dichas ocasiones el tratamiento quirúrgico abierto mediante la resección simple del polo distal puede ocasionar un colapso carpiano. El uso de la artroscopia para la resección del polo distal del escafoides permite, no solo la resección del polo distal a través de un abordaje mínimamente invasivo, sino también la evaluación y posible tratamiento de inestabilidades carpianas asociadas. En el presente trabajo se detalla la técnica quirúrgica para realizar la resección del polo distal del escafoides de forma artroscópica a través de tres portales artroscópicos.
\end{abstract}

Palabras clave: Artrosis escafo-trapecio-trapezoidea, resección distal del escafoides, artroscopia muñeca.

\begin{abstract}
Scapho-trapezium-trapezoid osteoarthritis is frequently associated with carpal instability. In these cases, an open distal scaphoid resection can produce a carpal collapse. The arthroscopic treatment for distal scaphoid resection allows, not only the resection of the distal pole, but also the diagnosis and treatment of associated carpal instabilities. In the current paper, the arthroscopic surgical technique for the resection of the distal pole of the scaphoid, through 3 arthroscopic portals, is explained.
\end{abstract}

Key words: Scaphotrapeziotrapezoid osteoarthritis, Distal scaphoid resection, wrist arthroscopy

La artrosis escafo-trapecio-trapezoidea (más conocida por sus siglas en inglés "STT") es la segunda artrosis más frecuente de la muñeca, esta descrita su presencia hasta en un 39\% de las exploraciones radiográficas asintomáticas y solo se debe tratar cuando la clínica está claramente correlacionada con los hallazgos de las pruebas de imagen 1,2, ya que en muchas ocasiones es un hallazgo radiográfico totalmente asintomático.

La artrosis STT puede estar asociada a inestabilidades carpianas. Por ello es importante la evaluación clara de dichas inestabilidades, valorando la insuficiencia o no de los ligamentos intracarpianos, la existencia de una subluxación dorsal del hueso grande y la lesión del ligamento escafolunar, con la aparición de una deformidad en extensión del segmento intercalado (DISI) (4) Figura I. Clásicamente, en estas situaciones la artroplastia de resección no estaba indicada ya que podía producir claramente un agravamiento de la inestabilidad carpiana y un colapso total en poco tiempo y el tratamiento de elección era la artrodesis escafo-trapecio-trapezoidea o la resección distal de escafoides con interposición tendinosa ${ }^{3}$.

Desde la existencia de la artroscopia de muñeca, varias indicaciones quirúrgicas han cambiado radicalmente en su enfoque, gracias a las posibilidades terapéuticas mínimamente invasivas de la artroscopia. Sin lugar a dudas, una de ellas ha sido la del tratamiento de la artrosis STT4,5, que permite realizar mínimas resecciones del polo distal del escafoides conservando la propiocepción y con la mínima desestabilización ligamentosa.

\section{INDICACIONES Y CONTRAINDICACIONES}

La indicación principal es la artrosis de la articulación escafo-trapecio-trapezoidea, pero puede extenderse a cualquier tratamiento exclusivo de esta zona como los gangliones intraóseos, la consolidación viciosa del polo distal del escafoides o los cuerpos libres articulares.
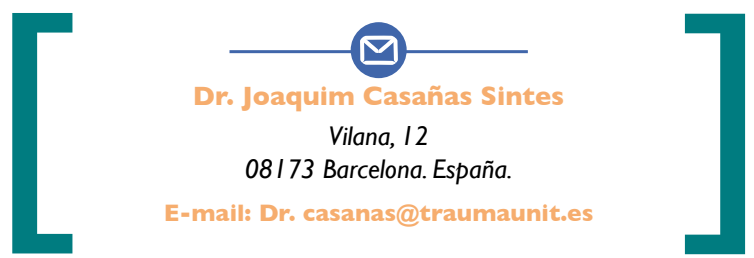

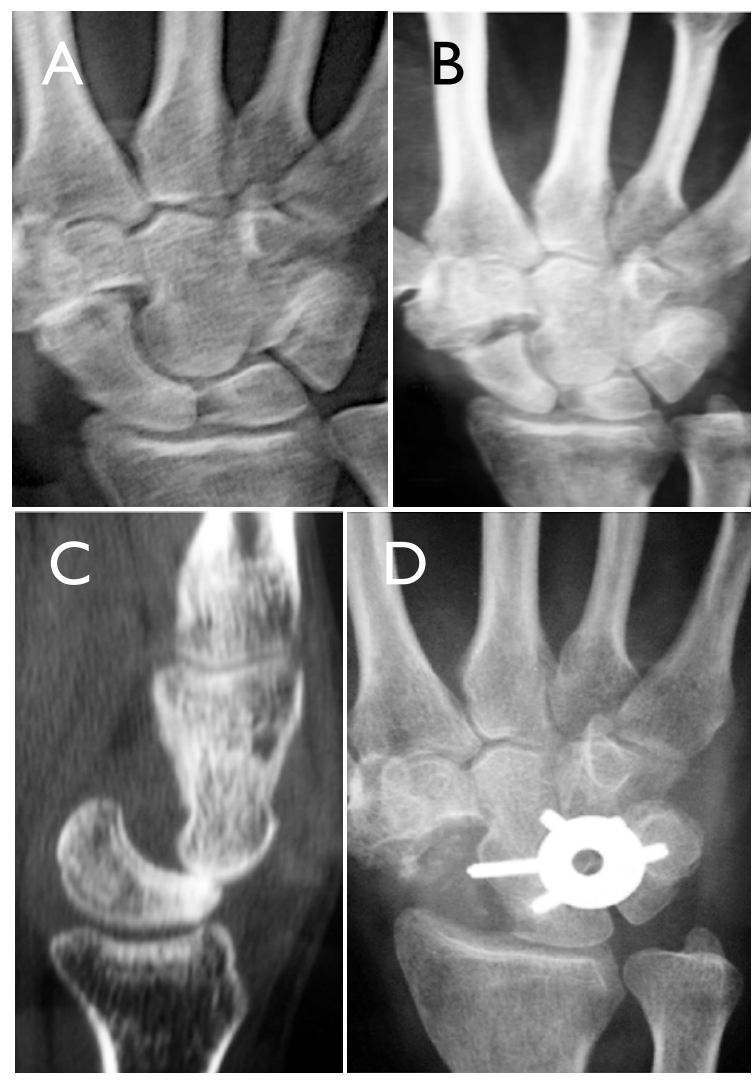

Figura I. A. Imagen característica de una artrosis STT, donde se adivina la sospecha de una disociación escafolunar. B. y C. Tras la resección abierta evolucionó a un colapso carpiano. D. Precisó posteriormente una artrodesis tipo cuatro esquinas.

En cuanto las contraindicaciones, además de las clásicas por existir problemas locales a nivel de los portales, se debe evitar el tratamiento artroscópico en presencia de transtornos vasculares y los pseudoaneurismas de la arteria radial.

Es importante considerar en este punto, que el tratamiento artroscópico será satisfactorio siempre y cuando el diagnóstico preoperatorio sea el adecuado y para ello es importante el evaluar siempre que nos encontramos con artropatías a este nivel, la ausencia de otras inestabilidades carpianas.

\section{ANATOMÍA QUIRÚRGICA}

Se identifica el portal mediocarpiano radial aproximadamente a 1 cm distal al portal 3-4, donde se notará una depresión proximal al hueso grande, en el espacio entre escafoides y el semilunar. Desde esta zona se ten-

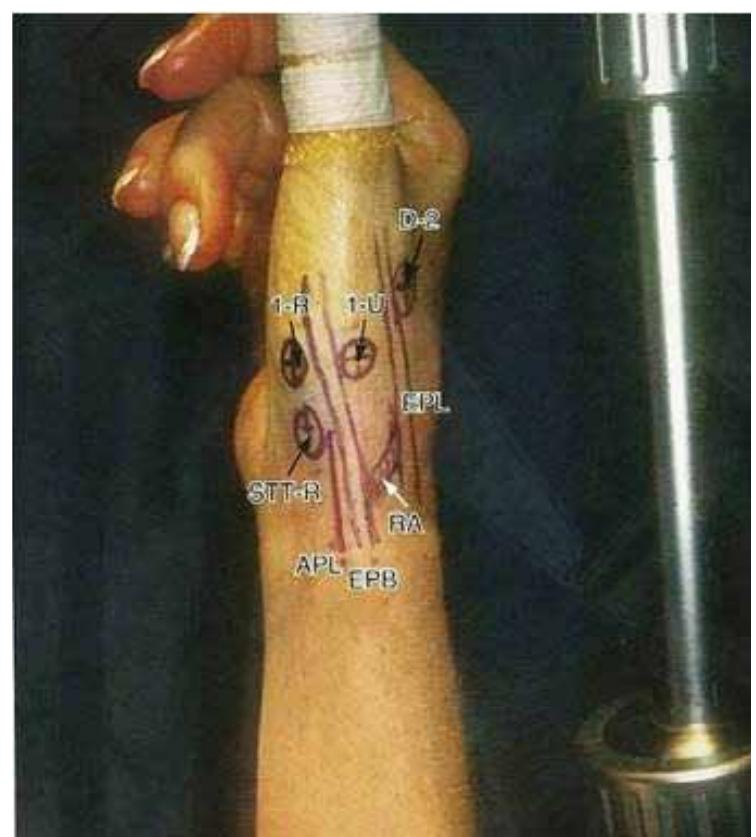

Figura 2. Se realiza tracción desde el pulgar y el segundo dedo para obtener una buena distensión de la columna radial de la muñeca. Se identifican los tendones del EPL, APB y FCR para marcar los portales MCR, STT dorsal y STT volar.

drá una buena visión de la continuación de ligamento radio-escafo-grande ${ }^{6,7}$, de la articulación escafolunar y de la articulación lunopiramidal.

Ascendiendo por el escafoides hacia su vertiente radial, se alcanzará fácilmente el polo de distal del escafoides, encontrando inicialmente, en la parte más superior, el trapezoide, y más a fondo, el aspecto volar del trapecio.

El portal STT dorsal se localiza en la porción más radial del tendón extensor pollicis longus (EPL), desde este portal se puede acceder de forma segura a la articulación STT. Se debe tener especial precaución en realizar los portales de forma muy superficial para no lesionar las ramas sensitivas del nervio radial. Algunos autores aconsejan realizar miniabordajes para identificar la rama recurrente de la arteria radial, aunque consideramos que si se realiza una meticulosa disección roma no es necesaria dicha maniobra ${ }^{8,9}$.

Para la práctica del portal STT volar ${ }^{10}$, se marca la línea del tendón del abductor pollicis brevis (APB) y practicaremos una incisión volar a él, en el espacio que queda entre el APB y el flexor carpi radialis (FCR). Puede servir de referencia el punto medio entre la estiloides radial y la base del primer metacarpiano (2) Figura 2. 


\section{TÉCNICA QUIRÚRGICA}

Se realiza la cirugía bajo bloqueo anestésico axilar y sedación, manguito de isquemia a una presión aproximada de $250 \mathrm{mmHg}$ y una cincha de anclaje en la mesa de mano operatoria como contra-tracción. En nuestra unidad se utiliza para esta intervención la torre de tracción de Whipple (Linvatec $(\mathbb{B})$ a 15 lb de tracción. Se modifica el eje de tracción habitual colocando los cazamuchachas en el primer y segundo dedo, para así poder disponer de mayor distracción sobre el primer radio y toda la columna radial del carpo. Se utiliza una óptica de $2.7 \mathrm{~mm}$, sinoviotomos de $2.9 \mathrm{~mm}$ para la sinovectomía y fresas de 2.9 ○ $3.5 \mathrm{~mm}$ para la resección distal del escafoides.

Se trata de una técnica de nivel intermedio-avanzado y por lo tanto, no se debe realizar si no se tiene cierta experiencia en artroscopia de muñeca. Asimismo es útil, al menos en los primeros casos, la realización de un control radiológico - Figura 3 tanto para la localización de la articulación STT, como para valorar el espacio obtenido tras la resección del polo distal del escafoides.

Se inicia la artroscopia por un portal mediocarpiano radial (MCR) para la visualización y exploración de la articulación mediocarpiana (2) Figura 4. En este momento se evaluará la inestabilidad del ligamento escafolunar y lunopiramidal. Esta evaluación de las posibles inestabilidades carpianas asociadas, previa a la resección del polo distal del escafoides, es una maniobra de gran importancia ya que servirá para decidir si es necesario o no su tratamiento después de la resección del escafoides.

Tras la exploración de la articulación mediocarpiana y con el artroscopio en el portal MCR, se dirige el artroscopio hacia el polo distal del escafoides. Se valora desde esta localización su margen dorsal y se procede a la práctica de un portal STT dorsal o cubital.

A través del portal STT dorsal se realiza un sinovectomía y desbridamiento que permita explorar la articulación y crear un buen campo de trabajo. A continuación se inicia el fresado con la pieza de $2.9 \mathrm{~mm}$ en la zona dorsal del polo distal del escafoides (1) Figura 5. Con la realización de este portal existe la posibilidad de lesión de estructuras tendinosas y vasculonerviosas, por lo que, como ya se ha mencionado, se debe tener especial cuidado en su establecimiento.

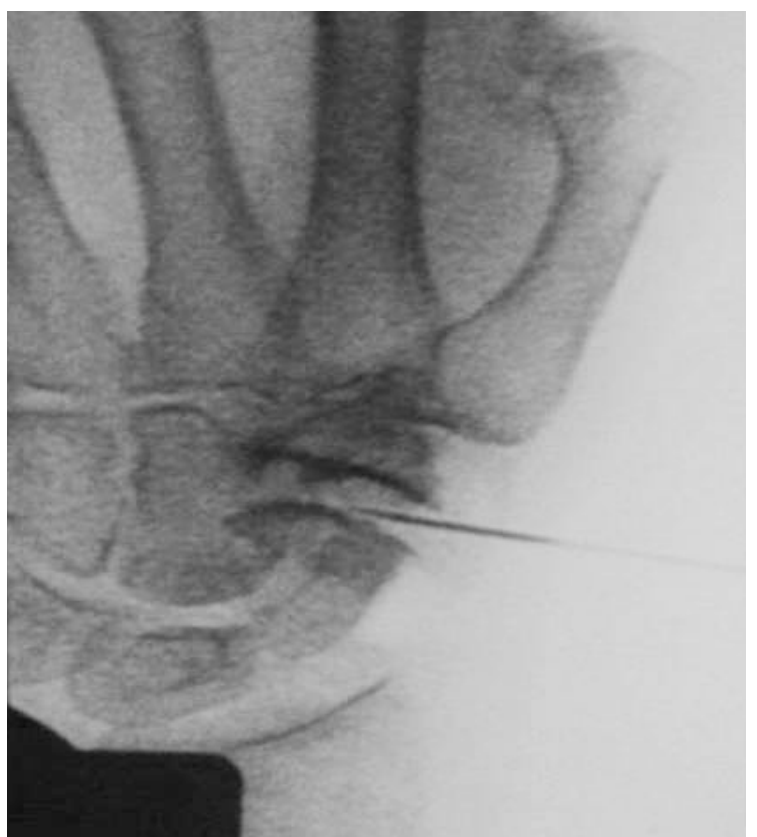

Figura 3. Al inicio de la práctica de esta técnica, es recomendable ayudarse de un control radiológico para marcar correctamente el portal y comprobar que tenemos una buena distracción de la articulación STT.

Posteriormente, se procede a practicar un portal STT radial o volar, que se ubica entre el APL y el FCR. La arteria radial y la rama recurrente volar circula debajo de dichas estructuras tendinosas, creando por tanto una zona segura de trabajo. Desde el portal radial se completa la resección más volar del escafoides, utilizándolo como portal de instrumentación, mientras se cambia el portal de visualización del portal MCR al STT dorsal.

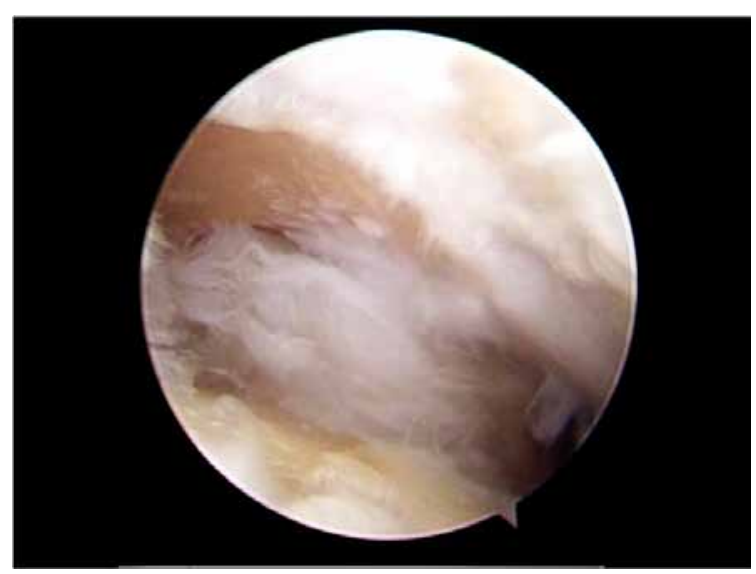

Figura 4. Imagen característica de la articulación STT con una osteoartritis evolucionada. Se observa una gran proliferación sinovial y el cartílago de las tres superficies totalmente denudado. 

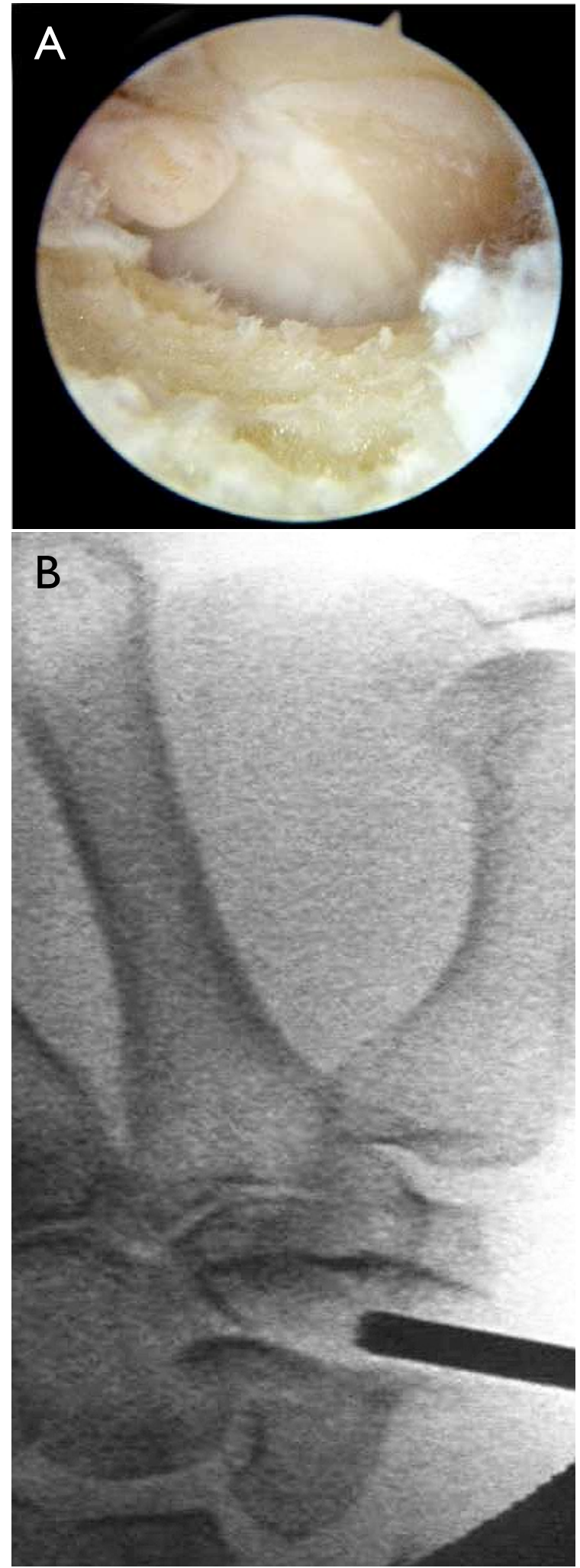

Figura 5. A. Visualización desde el portal STT dorsal de la resección realizada a nivel del polo distal del escafoides. B. Comprobación radiológica del espacio obtenido tras la resección artroscópica.
Si se está habituado en el manejo de esta articulación, se puede completar, de forma más rápida, la resección del polo distal del escafoides con la fresa de $3.5 \mathrm{~mm}$ en lugar de usar la de $2.9 \mathrm{~mm}$ (figura 5).

Finalmente, se realiza hemostasia si es necesario, se suelta la tracción y se comprueba que no exista impacto del extremo distal del escafoides con el extremo proximal del trapecio y del trapezoide. Se debe tener especial atención en comprobar la correcta resección del escafoides en el ángulo con el hueso grande, puesto que es el punto donde más fácilmente pueden quedar restos de polo distal escafoides que mantendrían la clínica de choque.

Se puede realizar un último control radiológico, relajando la tracción de la mano para estar seguros de haber obtenido una resección suficiente y completa de la superficie del polo distal de escafoides.

\section{MANEJO POSTOPERATORIO}

Se coloca un vendaje blando compresivo que permita movilización precoz de la extremidad y se pautan analgésicos y antiinflamatorios.

La recuperación funcional de la muñeca acostumbra a ser lenta y es normal que el paciente tarde un periodo mínimo de tres meses para encontrarse totalmente asintomático, recuperar un balance articular indoloro y una fuerza de prensión satisfactoria.

\section{COMPLICACIONES}

La más habitual es la persistencia de dolor a nivel de la zona radial de la muñeca pero que, a diferencia del cuadro preoperatorio que básicamente encontramos en flexión de la muñeca, persiste tanto en flexión como extensión. Esta clínica álgica acostumbra a resolverse de forma definitiva alrededor del tercer mes postoperatorio. Otra complicación frecuente es la resección insuficiente de la porción más cubital del polo distal, que puede producir un pinzamiento con el hueso grande $o$ la porción más volar del escafoides (4) Figura 6.

Otras posibles complicaciones que pueden aparecer al crear los portales pueden ser la lesión de las estructuras tendinosas vecinas y la lesión del arteria radial recurrente, lo que puede ocasionar un sangrado 


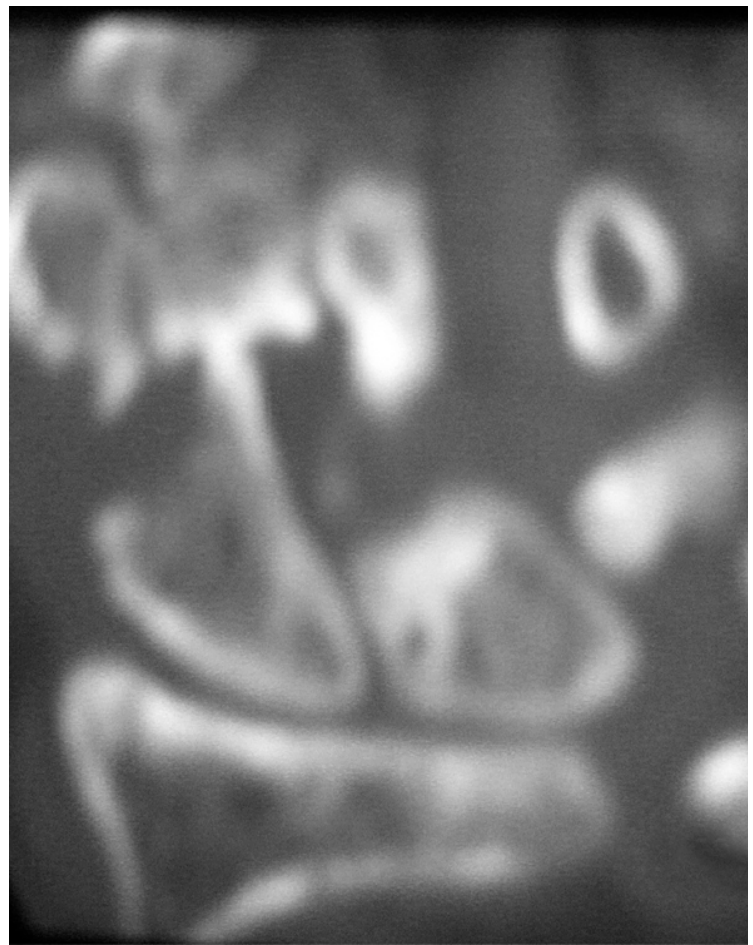

Figura 6. Imagen característica de una resección insuficiente de la porción más cubital del polo distal del escafoides, posible causa de mala evolución clínica.

importante al soltar la isquemia o el desarrollo de un pseudoaneurisma.

\section{CASO CLÍNICO}

Se trata de una paciente de 68 años que presentaba clínica de túnel carpiano asociada a dolor en la base del pulgar que se agravaba con la flexión de la muñeca y la compresión de la columna radial del pulgar. No presentaba dolor con las maniobras de inestabilidad del pulgar y retropulsión del mismo. La fuerza de pinza del pulgar preoperatoria era de 3 kilogramos.

Las exploraciones radiográficas demostraban la presencia de una artrosis STT sin otro signos de inestabilidad carpiana asodiados (aigura 7. La exploración física tampoco sugería ningún tipo de inestabilidad carpiana, con las maniobra de Reagan y Watson negativas.

El estudio neurofisiológico evidenciaba una compresión del nervio mediano a nivel del túnel carpiano y en la exploración la paciente presentaba una atrofia de la eminencia tenar.

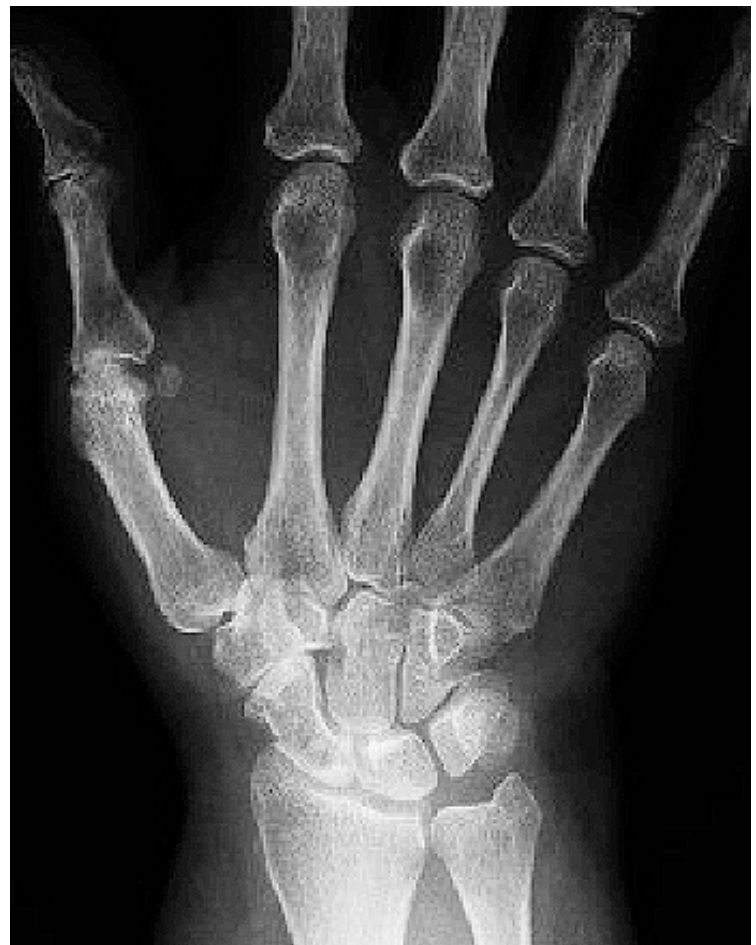

Figura 7. Imagen preoperatoria, con artrosis a nivel de la articulación STT sin otros signos radiológicos de inestabilidad carpiana.

Por todo ello, se realizó la descompresión del túnel carpiano mediante cirugía abierta y en el mismo acto quirúrgico se realizó, de forma artroscópica, la resección del polo distal del escafoides.

Se realizó inicialmente un portal mediocarpiano radial, comprobándose la ausencia de lesión escafolunar o lunopiramidal. Se procedió a la realización de un portal inicial STT dorsal desde donde se realizó una amplia sinovectomía para una correcta visualización del polo distal del escafoides y posteriormente una resección de la porción dorsal del polo distal del escafoides. Se completó la resección de la porción más volar a través del portal STT volar hasta obtener un suficiente margen de resección.

La paciente tuvo una evolución clínica sin complicaciones, con remisión completa del dolor a las seis semanas. Alcanzó una fuerza de pinza de $6 \mathrm{~kg}$ a las 12 semanas postoperatorias.

Las exploraciones radiográficas seriadas demuestran el mantenimiento del espacio creado a nivel del polo distal del escafoides @ Figura 8. La paciente se encuentra actualmente asintomática, a los 18 meses postoperatorios. 


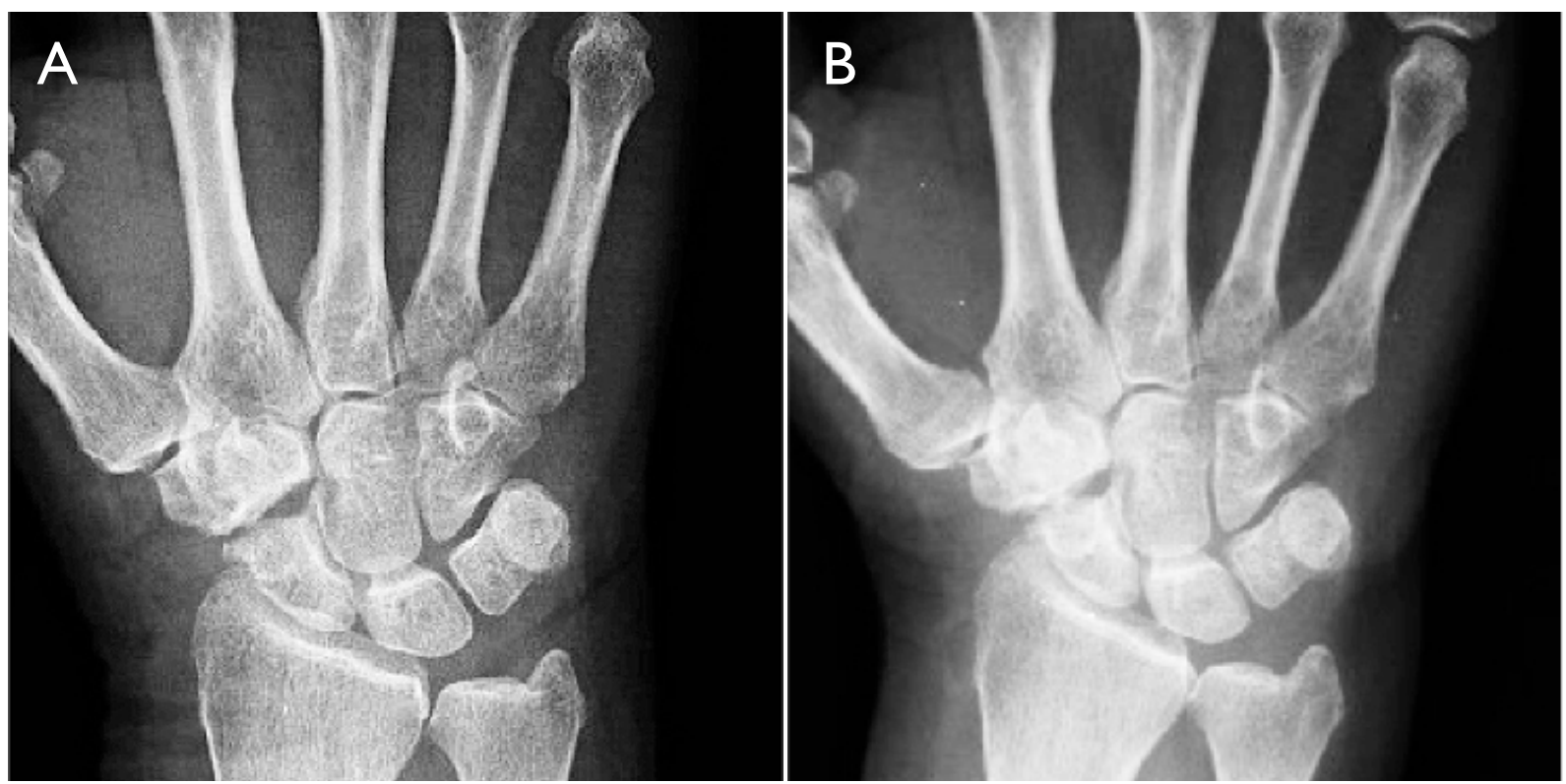

Figura 8. A. Imagen postoperatoria a las seis semanas de la intervención quirúrgica. B. Imagen postoperatoria a las doce semanas de la intervención quirúrgica Se evidencia una resección suficiente del polo distal del escafoides con ausencia de colapso carpiano, que se mantiene.

\section{CONFLICTOS DE INTERESES}

Los autores declaran no tener conflictos de intereses.

\section{BIBLIOGRAFÍA}

I. Moritomo H, Viegas SF, Nakamura K, Dasilva MF, Patterson RM. The scaphotrapezio-trapezoidal joint. Part I: An anatomic and radiographic study. J Hand Surg Am. 2000;25(5):899-910.

2. Viegas SF, Patterson RM, Hokanson JA, Davis J.Wrist anatomy: incidence, distribution, and correlation of anatomic variations, tears, and arthrosis. J. Hand Surg Am. 1993; I 8(3):463-75.

3. Mathoulin C, Darin F. Arthroscopic treatment of scaphotrapeziotrapezoid osteoarthritis. Hand Clin. $2011 ; 27(3): 319-22$
4. Normand J, Desmoineaux P, Boisrenoult P, Beaufils P.The arthroscopic distal pole resection of the scaphoid: clinical results in STT osteoarthritis. Chir Main. 20 I 2;3 I ( I): I3-7.

5. Garcia-Elias M. Excisional arthroplasty for scaphotrapeziotrapezoidal osteoarthritis. Hand Surg Am. 201 I;36(3):5 I 6-20.

6. Boden BP, Kozin SH, Berlet AC. Wrist arthroscopy. Am J Orthop. 1995;24(4):3 10-6.

7. Viegas SF. Midcarpal arthroscopy: anatomy and portals. Hand Clin. 1994; I0(4):577-87.

8. Botte MJ, Cooney WP, Linscheid RL. Arthroscopy of the wrist: anatomy and technique. J Hand Surg Am. 1989; | 4(2 Pt I):3 |3-6.

9. Abrams RA, Petersen M, Botte MJ. Arthroscopic portals of the wrist: an anatomic study. J Hand Surg Am. 1994; 19(6):940-4.

10. Baré J, Graham AJ, Tham SK. Scaphotrapezial joint arthroscopy: a palmar portal. J Hand Surg Am. 2003;28(4):605-9. 